\title{
Self-dynamic structure factor of dense liquids: Theory and simulation
}

\author{
Manel Canales \\ Departament de Física i Enginyeria Nuclear, Universitat Politècnica de Catalunya, B4-Campus Nord, 08034 Barcelona, Spain \\ Joan Àngel Padró \\ Departament de Física Fonamental, Universitat de Barcelona, Diagonal 647, 08028 Barcelona, Spain
}

(Received 29 June 2000; published 22 December 2000)

\begin{abstract}
The self-intermediate dynamic structure factor $F_{s}(k, t)$ of liquid lithium near the melting temperature is calculated by molecular dynamics. The results are compared with the predictions of several theoretical approaches, paying special attention to the Lovesey model and the Wahnström and Sjögren mode-coupling theory. To this end the results for the $F_{s}(k, t)$ second memory function predicted by both models are compared with the ones calculated from the simulations.
\end{abstract}

DOI: 10.1103/PhysRevE.63.011207

\section{INTRODUCTION}

The self-Van Hove distribution function $G_{s}(r, t)$ is a space- and time-dependent property normally used to study the single particle motions in liquids $[1,2] . G_{s}(r, t)$ can be evaluated from experimental data since its space and time Fourier transform is the well known self-dynamic-structurefactor $S_{s}(k, \omega)$, which can be measured by neutron scattering [1]. For simple dense liquids these data can be compared with the theoretical predictions obtained from different models $[3,4]$. However, under some circumstances (specially for small frequencies and large wavelengths) the experimental error can hinder the comparison. In these cases computer simulation [5] constitutes an alternative to experiment because on one hand the errors are smaller than those from experiments, and on the other it allows us to calculate more basic properties, which cannot be directly obtained from experiments.

In an earlier paper de Jong and co-workers [6] compared their neutron scattering $S_{s}(k, \omega)$ results for dense liquid lithium with the theoretical predictions obtained using several models. In particular they found that the Lovesey model [7] gave the best agreement with their experimental data. In this work we use the well known computer simulation technique of molecular dynamics (MD) [5] to calculate the selfintermediate scattering function $F_{s}(k, t)$, which is the space Fourier transform of $G_{s}(r, t)$. In fact, $F_{s}(k, t)$ is a more suitable quantity to test models because many of them are directly based on assumptions about $F_{s}(k, t)$. One example is the mode-coupling theory of Wahnström and Sjögren [8], who divided $F_{s}(k, t)$ into two parts: (1) a binary term that is associated with short time events and (2) a mode coupling term that incorporates the effects of more sophisticated processes that appear at longer times. The mode-coupling theory has been applied to calculate several properties of simple liquids, such as the velocity autocorrelation function $C(t)$ $[4,9]$, the shear viscosity coefficient $[10,11]$, the dynamic structure factors $[9,11-14]$, and the transverse current correlation functions [9]. In all cases the theoretical results showed a qualitative agreement with the simulation data.

In this work we compare the $F_{s}(k, t)$ data computed by MD for several representative wave numbers with the results obtained using the models described in the de Jong et al.
PACS number(s): 61.20.Gy, 61.20.Ja, 61.20.Lc, 61.25.Mv

work [6] as well as those calculated from mode-coupling theory. The main objective of this work is to check the reliability of the different theoretical approaches by comparing their predictions for a given potential with the corresponding " "exact' MD results. Moreover, the analysis of $F_{s}(k, t)$ and its memory function at different wave numbers will allow us to have a more complete picture of atomic motion in simple dense liquids. The study has been performed for liquid lithium and Lennard-Jones (LJ) fluids. However, we do not show the LJ results because they do not introduce any significant additional information. The paper is divided into five sections. In the first we define some basic quantities and describe the models and theories. Simulation details and the methodology used to compute $F_{s}(k, t)$ from the different models are described in the third section. Theoretical and experimental results are compared in the fourth section. Finally, the most relevant conclusions are summarized in Sec. V.

\section{THEORY}

\section{A. Basic definitions}

It is well known that the self-dynamic structure factor $S_{s}(k, \omega)$ is the space and time Fourier transform of the self Van Hove distribution function $G_{s}(r, t)$ [1]. However, from the theoretical point of view it is useful to define the selfintermediate scattering function $[2,3]$

$$
F_{s}(k, t)=\left\langle e^{-i \mathbf{k} \cdot[\mathbf{r}(t)-\mathbf{r}(0)]}\right\rangle,
$$

$\mathbf{r}(\mathrm{t})$ being the position of a particle and $\mathbf{k}$ the wave number.

Time correlation functions such as $F_{s}(k, t)$ can be studied through the formalism developed by Zwanzig and Mori, which is based on a Volterra integral equation $[2,4]$,

$$
\frac{d F_{s}(k, t)}{d t}=-\int_{0}^{t} M_{s}\left(k, t^{\prime}\right) F_{s}\left(k, t-t^{\prime}\right) d t^{\prime},
$$

$M_{s}(k, t)$ being the first memory function of $F_{s}(k, t)$. Similarly, the second memory function $N_{s}(k, t)$ is defined by

$$
\frac{d M_{s}(k, t)}{d t}=-\int_{0}^{t} N_{s}\left(k, t^{\prime}\right) M_{s}\left(k, t-t^{\prime}\right) d t^{\prime} .
$$


Two simple relations between the Laplace transforms of $F_{s}(k, t)$ and $N_{s}(k, t)$, which allow us to compute one function from the other, can be obtained by Laplace transforming Eqs. (2) and (3) [2,4],

$$
\begin{aligned}
& \widetilde{F}_{s}(k, z)=\left[-z+\frac{w_{0}^{2}}{-z+\widetilde{N}_{s}(k, z)}\right]^{-1}, \\
& \widetilde{N}_{s}(k, z)=z+w_{0}^{2}\left[z+\frac{1}{\widetilde{F}_{s}(k, z)}\right]^{-1},
\end{aligned}
$$

where $\omega_{0}^{2}=k_{B} T k^{2} / m . N_{s}(k, t)$ plays an important role in the study of single particle motions because many of the models or theories commonly used are based on that function [2-4].

\section{B. Models}

\section{Simple diffusion (SD)}

The well known simple diffusion model [2-4] assumes an exponential behavior of the self-intermediate scattering function,

$$
F_{s}^{S D}(k, t)=e^{-D k^{2} t},
$$

$D$ being the diffusion coefficient. This model, which constitutes a good approach in the range of large wavelengths (the so-called hydrodynamic limit), predicts a Lorentzian shape of the self-dynamic structure factor:

$$
S_{s}^{S D}(k, \omega)=\frac{1}{\pi} \frac{D k^{2}}{\omega^{2}+\left(D k^{2}\right)^{2}} .
$$

\section{2. de Schepper and Ernst (dSE) mode-coupling theory}

de Schepper and Ernst [15] proposed a mode-coupling correction to the simple diffusion model, which is calculated by taking into account only the coupling between the particle motion and the shear modes. Then $S_{s}^{d S E}(k, \omega)$ can be written as [16]

$$
S_{s}^{d S E}(k, \omega)=S_{s}^{S D}(k, \omega)+\frac{1}{\pi k k^{*} D} \operatorname{Re}\left[G\left(\frac{i \omega+D k^{2}}{\delta D k^{2}}\right)\right],
$$

with $k^{*}=16 \pi \beta m \rho D^{2}$ and $\beta=1 / k_{B} T$, where $\rho$ is the number density, $\delta=D /(D+\nu), \nu=\eta_{s} / m \rho$, and $\eta_{s}$ is the shear viscosity. The complex function $G(z)$ is given by

$$
G(z)=\arctan \left(\frac{1}{\sqrt{z-1}}\right)-\frac{(z-2) \sqrt{z-1}}{z^{2}} .
$$

The agreement between the theoretical results and the experimental data is particularly good for "hot" liquids and dense gases $[4,16]$.

\section{Nelkin-Ghatak model (NG)}

Nelkin and Ghatak [17] suggested a model obtained by solving a linearized Boltzmann equation. The self-scattering function is described by means of a probability integral with a complex argument,

$$
S_{s}^{N G}(k, \omega)=\frac{1}{\pi \alpha} \operatorname{Re}\left[\frac{\bar{z}}{1-\bar{z}}\right],
$$

where $\bar{z}=\sqrt{\pi} y w(z) . w(z)$ is the probability integral for a complex argument, which is a tabulated function [18]. $z=x$ $+i y, x=-\omega /\left(\sqrt{2} k v_{0}\right), y=\alpha /\left(\sqrt{2} k v_{0}\right), v_{0}^{2}=k_{B} T / m$, and $\alpha$ is the averaged collision frequency, which in the small $k$ limit is $\alpha=v_{0}^{2} / D$. This model gives the correct behavior in the limit of both small and large $k$ values.

\section{Lovesey (LO) model}

The Lovesey model [7] assumes an exponential decay of the second memory function

$$
N_{s}^{L O}(k, t)=\left[2 \omega_{0}^{2}+\Omega_{0}^{2}\right] e^{-t / \tau_{L}(k)},
$$

with $\omega_{0}^{2}=k_{B} T k^{2} / m$ and $\Omega_{0}^{2}$ being the square of the Einstein frequency, which can be obtained from the radial distribution function $g(r)$ and the pair potential $\phi(r)$ [4]. $\tau_{L}(k)$ is a $k$ dependent collision time. In this work we have used the approach proposed by de Jong et al. [6]: $\tau_{L}(k)$ $=k_{B} T /\left[m D\left(2 \omega_{0}^{2}+\Omega_{0}^{2}\right)\right]$. One of the advantages of the Lovesey model is that $S_{s}^{L O}(k, \omega)$ has an analytical form. Moreover, a good agreement was observed between its predictions and some experimental data [4].

\section{Wahnström and Sjögren (WS) mode-coupling theory}

As mentioned above, the de Schepper and Ernst theory takes into account only the coupling of the particle motion with the shear modes. That is why this theory fails for liquids near the melting point, just when the couplings to other quantities are not negligible [4]. Wahnström and Sjögren (WS) $[8,13]$ developed a more general theory that includes the couplings to both density and shear modes. To sum up they divided the second memory function $N_{s}^{W S}(k, t)$ into two parts: a binary term $N_{s B}(k, t)$, which is associated with the short time dynamics, and a mode-coupling term $N_{s M C}(k, t)$, which incorporates more sophisticated events that appear at longer times. The calculation of this last contribution is based on the idea that the motion of a tagged particle is influenced by the constraints collectively imposed by its neighboring particles [4]:

$$
N_{s}^{W S}(k, t)=N_{s B}(k, t)+N_{s M C}(k, t) .
$$

Wahnström and Sjögren divided the mode-coupling contribution into four parts, which reflect, respectively, the couplings of a moving atom to the density and the longitudinal and transverse (shear) currents of the system $[8,13]$,

$$
N_{s M C}(k, t)=N_{s 00}(k, t)+N_{s 01}(k, t)+N_{s 11}(k, t)+N_{s 22}(k, t) .
$$


The Laplace transforms of these terms are

$$
\begin{gathered}
\widetilde{N}_{s 00}(k, z)=\widetilde{R}_{s 00}(k, z), \\
\widetilde{N}_{s 01}(k, z)=\widetilde{R}_{s 01}(k, z)\left[\widetilde{N}_{s B}(k, z)+\widetilde{N}_{s}^{W S}(k, z)\right], \\
\widetilde{N}_{s 11}(k, z)=\widetilde{N}_{s B}(k, z) \widetilde{R}_{s 11}(k, z) \widetilde{N}_{s}^{W S}(k, z),
\end{gathered}
$$

$$
\begin{aligned}
\widetilde{N}_{s 22}(k, z)= & {\left[\widetilde{N}_{s B}(k, z)+\widetilde{R}_{s 00}(k, z)+\widetilde{N}_{s B}(k, z) \widetilde{R}_{s 01}(k, z)\right] } \\
& \times \widetilde{R}_{s 22}(k, z) \widetilde{N}_{s}^{W S}(k, z) .
\end{aligned}
$$

The Laplace transform of the total second memory function can be written as

$$
\widetilde{N}_{s}^{W S}(k, z)=\frac{\widetilde{N}_{s}^{W S^{\prime}}(k, z)}{1-\widetilde{R}_{s 01}(k, z)-\widetilde{N}_{s B}(k, z) \widetilde{R}_{s 11}(k, z)-\widetilde{N}_{s}^{W S^{\prime}}(k, z) \widetilde{R}_{s 22}(k, z)},
$$

where

$$
\begin{aligned}
\widetilde{N}_{s}^{W S^{\prime}}(k, z)= & \widetilde{N}_{s B}(k, z)+\widetilde{R}_{s 00}(k, z)+\widetilde{N}_{s B}(k, z) \\
& \times \widetilde{R}_{s 01}(k, z) .
\end{aligned}
$$

$\widetilde{R}_{s i j}(k, z)$ are the Laplace transforms of the recollision terms $R_{s i j}(k, t)$ which are compiled in Refs. $[8,13]$. The final form, when spherical symmetry is assumed, is

$$
\begin{aligned}
s R_{s 00}(k, t)= & \frac{\rho k_{B} T}{4 \pi^{2} m} \int_{0}^{\pi} \Delta F_{s}\left(k, k^{\prime}, t\right) \cos ^{2} \theta \sin \theta d \theta \\
& \times \int_{0}^{\infty} k^{\prime 4} c^{2}\left(k^{\prime}\right) F\left(k^{\prime}, t\right) d k^{\prime}, \\
R_{s 01}(k, t)= & -\frac{1}{4 \pi^{2} \Omega_{0}^{2}} \int_{0}^{\pi} \Delta F_{s}\left(k, k^{\prime}, t\right) \cos ^{2} \theta \sin \theta d \theta \\
& \times \int_{0}^{\infty} k^{\prime 2} c\left(k^{\prime}\right)\left[\gamma_{L}\left(k^{\prime}\right)+\frac{\rho k_{B} T}{m} k^{\prime 2} c\left(k^{\prime}\right)\right] \\
& \times \frac{\partial F\left(k^{\prime}, t\right)}{\partial t} d k^{\prime}, \\
R_{s 11}(k, t)= & -\frac{1}{4 \pi^{2} \rho \Omega_{0}^{4}} \int_{0}^{\pi} \Delta F_{s}\left(k, k^{\prime}, t\right) \cos ^{2} \theta \sin \theta d \theta \\
& \times \int_{0}^{\infty} k^{\prime 2}\left[\gamma_{L}\left(k^{\prime}\right)+\frac{\rho k_{B} T}{m} k^{\prime 2} c\left(k^{\prime}\right)\right]^{2} \\
& \times \frac{C_{l}\left(k^{\prime}, t\right)}{C_{l}\left(k^{\prime}, 0\right)} d k^{\prime}, \\
R_{s 22}(k, t)=- & \frac{1}{4 \pi^{2} \rho \Omega_{0}^{4}} \int_{0}^{\pi} \Delta F_{s}\left(k, k^{\prime}, t\right)\left(1-\cos ^{2} \theta\right) \sin \theta d \theta \\
\times & \int_{0}^{\infty} k^{\prime 2}\left[\gamma_{T}\left(k^{\prime}\right)\right]^{2} \frac{C_{t}\left(k^{\prime}, t\right)}{C_{t}\left(k^{\prime}, 0\right)} d k^{\prime},
\end{aligned}
$$

where

$$
\begin{aligned}
\Delta F_{s}\left(k, k^{\prime}, t\right)= & F_{s}\left(\sqrt{k^{2}+k^{\prime 2}-2 k k^{\prime} \cos \theta}, t\right) \\
& -F_{0}\left(\sqrt{k^{2}+k^{\prime 2}-2 k k^{\prime} \cos \theta}, t\right),
\end{aligned}
$$

$\rho$ is the density, $c(k)$ is the Fourier transform of the direct correlation function, and $\gamma_{L}(k)$ and $\gamma_{T}(k)$ are two $k$-dependent quantities that are defined in Refs. $[4,19]$. $F(k, t)$ is the intermediate coherent scattering function, $C_{l}(k, t)$ and $C_{t}(k, t)$ are, respectively, the longitudinal and transverse current correlation functions $[2,4]$, and $F_{0}(k, t)$ $=e^{-\omega_{0}^{2} t^{2} / 2}$ is the free particle limit form of the intermediate scattering function.

$N_{s 00}(k, t)$, which is in general the dominant term, starts as $t^{4}$ and incorporates the effects of the coupling of the velocity of a tagged particle with the density fluctuations of the surrounding medium. The $N_{s 01}(k, t), N_{s 11}(k, t)$, and $N_{s 22}(k, t)$ contributions reflect, respectively, the coupling with the first derivative of the dynamic structure factor and with the longitudinal and transverse currents $[8,13]$.

In this study we will assume two different models for the binary term. The first is based on a Gaussian approach to the memory function and the second is obtained by solving a kinetic equation. Both treatments give the same result at small $k$ 's, but produce important discrepancies at large $k$ 's.

a. Gaussian binary term approach. It is well known that the short time behavior of the velocity autocorrelation memory function $K(t)$ is well described by a Gaussian function $[4,20]$. Due to the close relation between $N_{s}(k, t)$ and $K(t)[2,4]$, some authors have also assumed a Gaussian decay for $N_{s B}(k, t)[4]$,

$$
N_{s B}^{G a u}(k, t)=\left[2 \omega_{0}^{2}+\Omega_{0}^{2}\right] e^{-t^{2} / \tau_{G}^{2}(k)},
$$

$\tau_{G}(k)$ being [14]

$$
\tau_{G}(k)=\frac{\tau_{0}}{\sqrt{1+\frac{5}{2} \omega_{0}^{2} \tau_{0}^{2}}} .
$$


$\tau_{0}$ plays the role of an averaged collision time, which can be calculated in terms of $g(r)$ and the first and second derivatives of $\phi(r)$ [4]. Some authors also assumed a functional form with a softer decay, such as $\operatorname{sech}^{2}\left[t / \tau_{G}(k)\right][4,12,21]$. However, we carried out a careful analysis of both Ansätze that has shown that the short time dynamics is better described by a Gaussian function. Similar conclusions were found in a previous study where the mode-coupling predictions for $K(t)$ were compared with the simulation data [20].

b. Kinetic equation binary term approach. Wahnström and Sjogren [8] used a binary term that was obtained by solving a kinetic equation [22-24] for the binary part $F_{s B}(k, t)$ of the self intermediate scattering function. The solution of this equation has the following analytical form $[8,13]$ :

$\widetilde{F}_{s B}(k, z)=\frac{1}{\widetilde{\Gamma}(k, z)} \sum_{n=0}^{\infty} \frac{\left[\tilde{\kappa}^{2}(k, z)\right]^{n}}{\tilde{\rho}(k, z)[\tilde{\rho}(k, z)+1] \cdots[\tilde{\rho}(k, z)+n]}$,

$\tilde{\kappa}^{2}(k, z)$ and $\tilde{\rho}(k, z)$ being

$$
\begin{gathered}
\tilde{\kappa}^{2}(k, z)=\frac{w_{0}^{2}}{[\widetilde{\Gamma}(k, z)]^{2}}, \\
\tilde{\rho}(k, z)=\tilde{\kappa}^{2}(k, z)-\frac{z}{\widetilde{\Gamma}(k, z)} .
\end{gathered}
$$

$\widetilde{\Gamma}(k, z)$ is the Laplace transform of a generalized friction coefficient, which is assumed to decay following a Gaussian functional form $[8,13]$,

$$
\Gamma(k, t)=\Omega_{0}^{2} e^{-t^{2} / \tau_{G}^{2}(k)},
$$

where the $k$-dependent time parameter $\tau_{G}(k)$ is calculated from Eq. (17). Therefore $\widetilde{F}_{s B}^{\text {Kin }}(k, z)$ is obtained using Eqs. (18)-(21), and the so-called kinetic binary part of the second memory function $N_{s B}^{K i n}(k, t)$ is calculated using Eq. (5).

\section{COMPUTATIONAL PROCEDURE}

\section{A. Simulation details}

The results presented in this paper were obtained from MD simulations of particles with the mass of ${ }^{7} \mathrm{Li}$ by assuming an effective potential with no adjustable parameters deduced from the neutral pseudoatom (NPA) method [25]. The thermodynamic conditions are $T=470 \mathrm{~K}$ and $\rho$ $=0.044512 \AA^{-3}$, which correspond to liquid lithium close to the melting point. A cubic box with 1750 particles and periodic boundary conditions [5] were considered. Beeman's algorithm [26] with a time step of 3 fs was used for the numerical integration of the motion equations. After an equilibration period of $10^{4}$ time steps, a run of $10^{5}$ configurations was carried out to compute the incoherent intermediate scattering functions $F_{s}(k, t)$. Besides the radial distribution function $g(r)$, the mean square displacement $r^{2}(t)$, the shear viscosity $\eta_{s}$, and some $k$-dependent properties, includ-

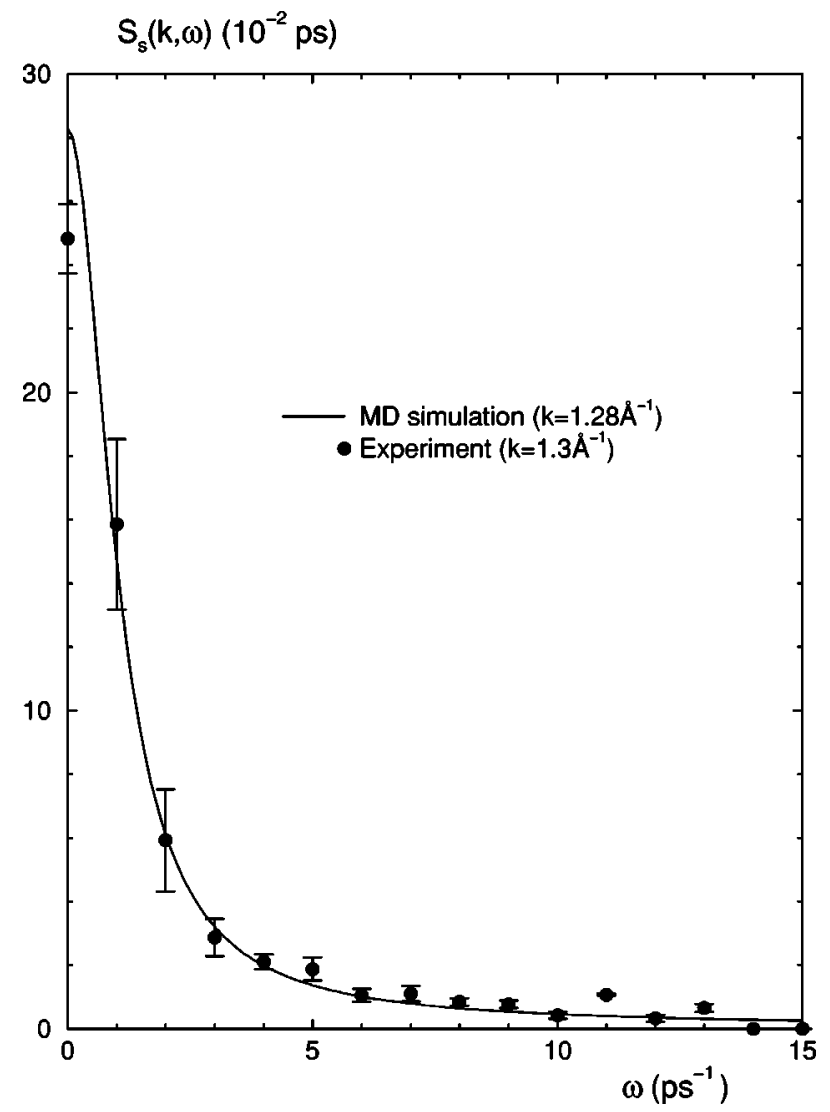

FIG. 1. Experimental [12] and MD results for the self dynamic structure factor.

ing the coherent intermediate scattering functions $F(k, t)$, the longitudinal $C_{l}(k, t)$ and transverse $C_{t}(k, t)$ current correlation functions were calculated. The $k$-dependent properties were determined for 20 different $k$ 's within the $0.185 \AA^{-1}$ and $5 \AA^{-1}$ interval.

In previous simulations $[27,28]$ it was shown that the properties of liquid ${ }^{7} \mathrm{Li}$ calculated with the NPA potential agree with the available experimental data. Moreover, subsequent measurements of the dynamic structure factor $S(k, \omega)$ by inelastic X-ray scattering [29-31] have also corroborated the good agreement between the experimental and simulation data. The self dynamic structure factor $S_{s}(k, \omega)$ calculated from the $F_{s}(k, t) \mathrm{MD}$ data at $k=1.28 \AA^{-1}$ is plotted in Fig. 1. This result is in good agreement with the inelastic neutron scattering measurements at $k=1.3 \AA^{-1}$ [12] which are also displayed in Fig. 1. In relation to these measurements it should be noted that the neutron scattering spectrum for liquid lithium is proportional to a weighted sum of the coherent $S(k, \omega)$ and the incoherent $S_{s}(k, \omega)$ dynamic structure factors $[1,6]$. So, in general, it is difficult to calculate $S_{s}(k, \omega)$. However, at sufficiently small $k$ 's, the incoherent term is greatly dominant. For instance, in the case of liquid lithium at $k=1.3 \AA^{-1}$, the incoherent term is more than two orders of magnitude higher than the coherent contribution.

\section{B. Calculation of $F_{s}(k, t)$ and $N_{s}(k, t)$}

The MD results for the intermediate scattering function $F_{s}^{M D}(k, t)$ and the corresponding second memory function 
$N_{s}^{M D}(k, t)$ were chosen as the "exact" or reference data. $N_{s}^{M D}(k, t)$ was computed from $F_{s}^{M D}(k, t)$ by solving Eqs. (2) and (3) according to the Berne and Harp method [32].

Theoretical results were obtained using the equations in Sec. II B. The required values of $D, \eta_{s}$, and $g(r)$ were taken from the MD results in Refs. [27,28]. $F_{s}^{S D}(k, t)$ was computed according to Eq. (6). $F_{s}^{d S E}(k, t)$ and $F_{s}^{N G}(k, t)$ were calculated from Eqs. (8) and (9), respectively. $F_{s}^{L O}(k, t)$ were obtained using Eqs. (4) and (10). The computation of $F_{s}^{W S}(k, t)$ or $N_{s}^{W S}(k, t)$ is more laborious and requires the previous calculation of $N_{s M C}(k, t)$ and $N_{s B}(k, t)$.

The $\Omega_{0}$ and $\tau_{0}$ parameters of the binary terms $N_{s B}^{\text {Gau }}(k, t)$ and $N_{s B}^{K i n}(k, t)$ were computed from $g(r)$ according to the procedure described in Ref. [20]. It should be noted that the static structure factor $S(k)$, which is required for the computation of $\tau_{0}$, was obtained by extending $g(r)$ to distances greater than the half length of the simulated cubic box using the procedure proposed by Verlet $[5,33]$. To this end the Ornstein-Zernicke equation was solved using the algorithm designed by Zerah [34]. The same procedure was used to calculate the Fourier transform of the direct correlation function $c(k)$, which is also required for the computation of $N_{s M C}(k, t)$.

Knowledge of the $F(k, t), F_{s}(k, t), C_{l}(k, t)$, and $C_{t}(k, t)$ functions for all wave numbers is needed to compute $N_{s M C}(k, t)$ [see Eqs. (12)-(15)]. However, MD simulation allows calculation of these functions only for a finite set of $k$ 's compatible with the periodic boundary conditions. For this reason we considered four $k$ regions and different treatments were used for each of them.

For $k<0.185 \AA^{-1}$ the hydrodynamic model was assumed. The thermodynamic and transport coefficients required to compute these functions were obtained from the energy and pressure fluctuations [5] and using the GreenKubo relations [5,35], respectively.

An interpolation procedure [36] was been applied to the MD results to obtain the correlation functions within the $0.185 \AA^{-1} \leqslant k \leqslant 2 \AA^{-1}$ interval.

In the $2 \AA^{-1} \leqslant k \leqslant 5 \AA^{-1}$ region, the functions change sharply and interpolation methods cannot be used. Thus, the viscoelastic model developed by Lovesey [37] was assumed. It is based on the assumption of an exponential behavior of the second order memory function of $F(k, t)[4,37]$. The coefficients appearing in the $F(k, t)$ expression can be obtained from the radial distribution function, the pair potential, and its derivatives. This model has been checked for different simple liquids [4,37-39] and in all cases it has rendered good results for $k$ 's close to the $S(k)$ maximum. The viscoelastic model for the transverse current correlation function can be constructed by an analogous procedure, but in this case the knowledge of the shear viscosity coefficient $\eta_{s}$ and the assumption of a special closure relation, such as the Akcasu and Daniels approach, are required [4]. $F_{s}(k, t)$ has been computed by assuming a Gaussian approximation $[3,4]$ $F_{s}(k, t)=e^{-k^{2} r^{2}(t) / 6}$, where $r^{2}(t)$ is the mean square displacement.

For $k>5 \AA^{-1}$ the free particle limit approach $[2,4]$ was assumed. Thus,

$$
F(k, t)=F_{s}(k, t)=C_{t}(k, t) / C_{t}(k, 0)=F_{0}(k, t)=e^{-\omega_{0}^{2} t^{2} / 2},
$$

and

$$
C_{l}(k, t) / C_{l}(k, 0)=\left(1-\omega_{0}^{2} t^{2}\right) e^{-\omega_{0} t^{2} / 2} .
$$

\section{RESULTS}

\section{A. Binary term}

Both the "kinetic" $N_{s B}^{K i n}(k, t)$ and the "Gaussian", $N_{s B}^{G a u}(k, t)$ approaches have been adopted by several authors to compute $N_{s}^{W S}(k, t)$. The "kinetic" Ansatz was used by Wahnström and Sjögren to study liquid argon and rubidium [8], by Gudowski et al. in their analysis of liquid lead [13], and by Shimojo and co-workers to study sodium near its triple point [14]. The "Gaussian" approach is described in Balucani and Zoppi [4]. However, to our knowledge, there has been no a comparison of the reliability of the two approaches. In this work both Ansätze are compared with the "exact" binary term $N_{s B}^{M D}(k, t)$. This was evaluated according to Eq. (11) by subtracting from $N_{s}^{M D}(k, t)$ the theoretical mode-coupling contribution $N_{s M C}(k, t)$ calculated according to the procedure described in Sec. III B:

$$
N_{s B}^{M D}(k, t)=N_{s}^{M D}(k, t)-N_{s M C}(k, t) .
$$

The resulting $N_{s B}^{G a u}(k, t), N_{s B}^{K i n}(k, t)$, and $N_{s}^{M D}(k, t)$ are monitored in Fig. 2 for small $\left(0.18 \AA^{-1}\right)$, intermediate $\left(1.28 \AA^{-1}\right)$, and large $\left(4.18 \AA^{-1}\right) k$ 's. The results for a wave number $\left(2.56 \AA^{-1}\right)$ close to the $k$ value where $S(k)$ reaches its first maximum $\left(k_{m}=2.50 \AA^{-1}\right)$ [27] are also displayed. All the functions in Fig. 2 have been normalized by the corresponding $N_{s}^{M D}(k, 0)$ value. At small $k$ 's both models give the same result. However, for high $k$ 's, $N_{s B}^{K i n}(k, t)$ exhibits some damped oscillations with a clear minimum, which is deeper and appears at shorter times as $k$ increases. In contrast $N_{s B}^{M D}(k, t)$ shows only a shallow minimum at the largest $k$ value $\left(4.18 \AA^{-1}\right)$. Similar discrepancies between simulation findings and theoretical results obtained using the "kinetic" approach were also observed by Gudowski et al. for liquid lead [13] and by Shimojo and co-workers for liquid sodium [14]. It is important to note that, in the free particle limit, the second memory function calculated assuming $F_{s}(k, t)=e^{-\omega_{0}^{2} t^{2} / 2}$ is similar to $N_{s B}^{\text {Kin }}(k, t)$. This is not casual because the "kinetic" approach satisfies both the hydrodynamic and the free particle limits [23]. $N_{s B}^{M D}(k, t)$ for large $k$ 's exhibits a minimum after the initial decay that can be associated with the free particle limit behavior. Even though the "Gaussian" Ansatz does not give rise to this minimum, Fig. 2 shows that $N_{s B}^{\text {Gau }}(k, t)$ fits the MD data better than $N_{s B}^{K i n}(k, t)$. Thus, in this work we have computed $N_{s}^{W S}(k, t)$ using the "Gaussian" approximation.

\section{B. Self-intermediate scattering function}

The $F_{s}(k, t)$ results from MD are compared with the SD, dSE, NG, LO, and WS theoretical predictions in Fig. 3. 

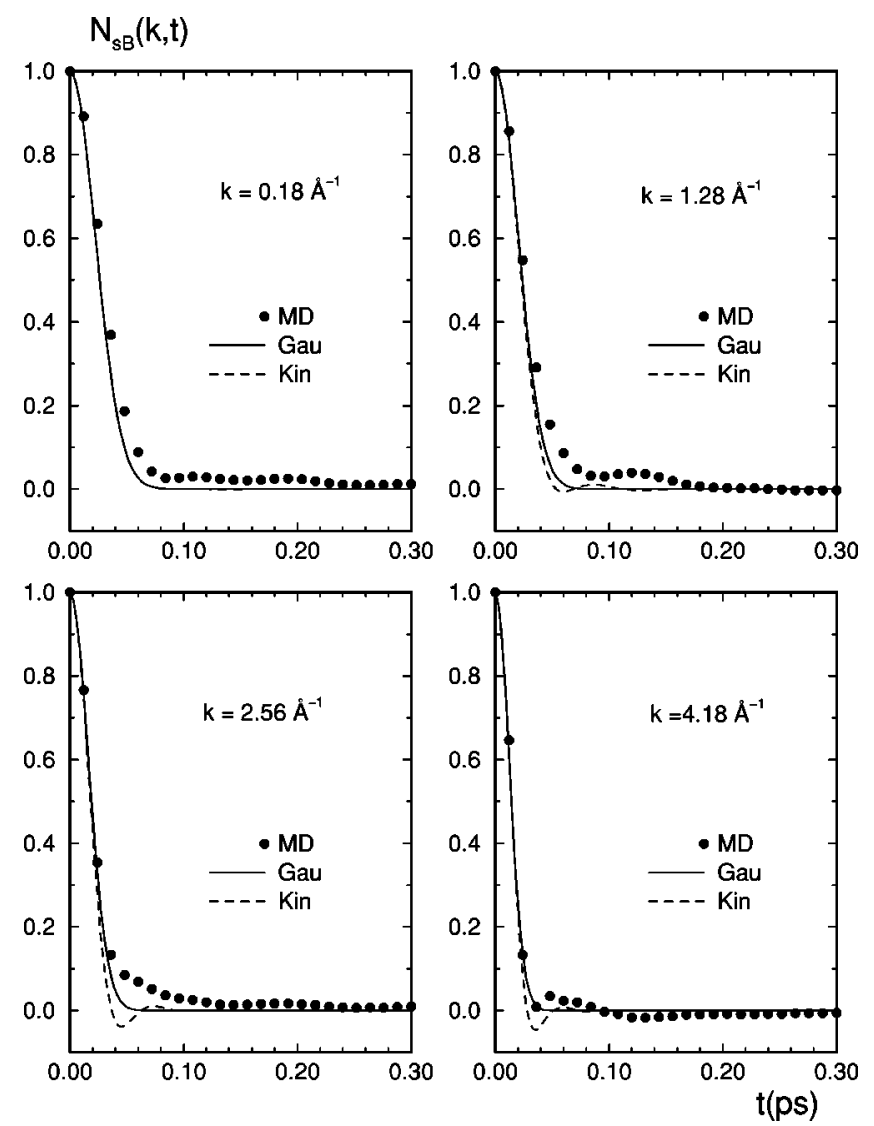

FIG. 2. Normalized binary term of the second memory function of the self dynamic structure factor $F_{s}(k, t)$. Simulation data (MD), Gaussian model (Gau) [4], and Kinetic model (Kin) [8].

$F_{s}^{M D}(k, t)$ shows the characteristic monotonic time decay that becomes faster as $k$ increases. $F_{s}^{L O}(k, t)$ and $F_{s}^{S D}(k, t)$ agree with the MD data for small $\left(0.18 \AA^{-1}\right)$ and intermediate $\left(1.28 \AA^{-1}\right)$ wave numbers. However, important discrepancies, which of course are more evident for the SD model, are observed at larger $k$ values $\left(2.56 \AA^{-1}\right.$ and $\left.4.18 \AA^{-1}\right)$. Moreover, both $F_{s}^{N G}(k, t)$ and $F_{s}^{d S E}(k, t)$ are in marked desagreement with $F_{s}^{M D}(k, t)$, the discrepancies being even larger than for $F_{s}^{S D}(k, t)$. These results confirm the conclusions of de Jong and co-workers [6], who compared the $S_{s}(k, \omega)$ factors calculated using the SD, LO, NG, and dSE models with their neutron scattering measurements. In particular, they stated that the LO model gives the best agreement with the experimental data. At very short times $(t<0.1 \mathrm{ps}) F_{s}^{L O}(k, t)$ and $F_{s}^{W S}(k, t)$ are in excellent agreement with $F_{s}^{M D}(k, t)$. This result can be explained by considering the short time expansion $F_{s}(k, t) \simeq 1-\frac{1}{2} \omega_{0}^{2} t^{2}[3]$ and bearing in mind that both $S_{s}^{L O}(k, \omega)$ and $S_{s}^{W S}(k, \omega)$ satisfy the three first moment conditions. However, at larger times $(t>0.1 \mathrm{ps})$ the Lovesey model shows a nonrealistic oscillatory behavior for all wave numbers, which is more obvious at high $k$ 's (see the results for $k=2.5 \AA^{-1}$ and $k$ $=4.18 \AA^{-1}$ ). These oscillations give rise to the presence of a spurious relative maximum in the $S_{s}^{L O}(k, \omega)$ spectra at high frequencies (e.g., at $\omega=60 \mathrm{ps}^{-1}$ for $k=4.18 \AA^{-1}$ ). Noticeable discrepancies between the WS predictions and the simu-
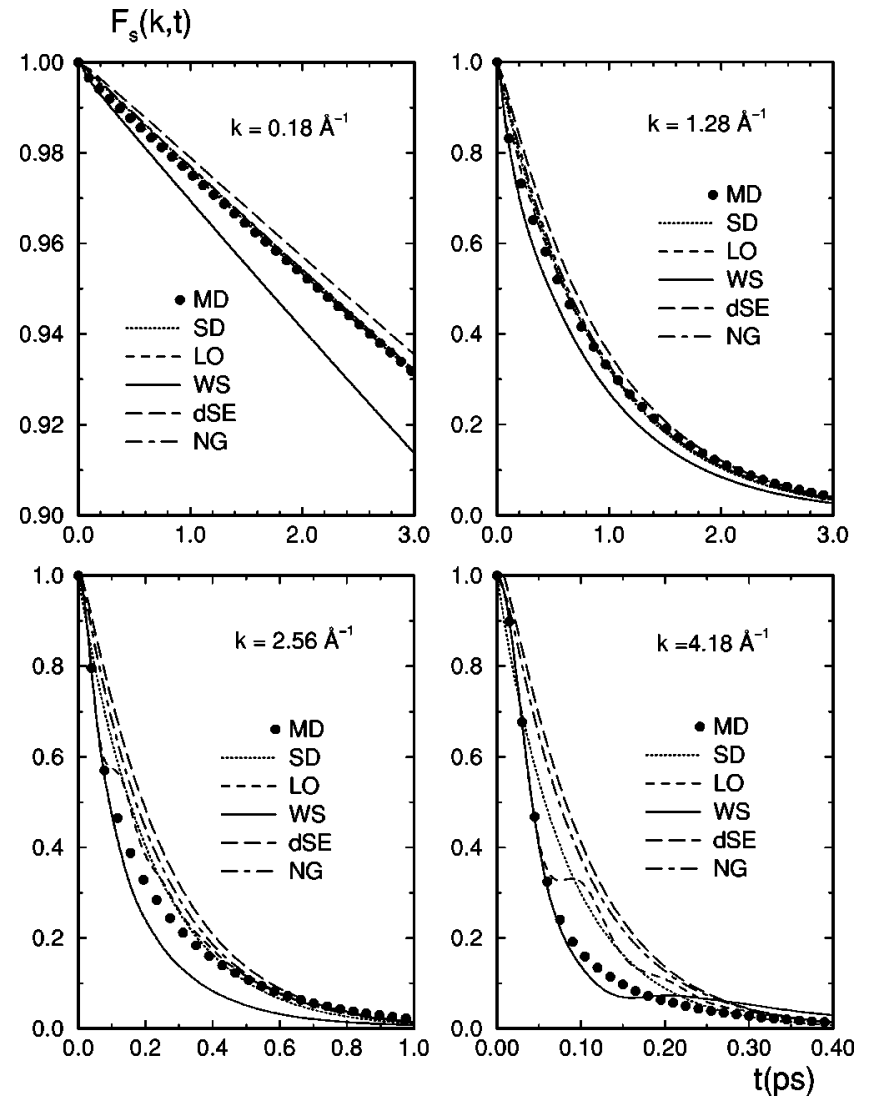

FIG. 3. Self dynamic structure factor. Simulation results (MD), simple diffusion model (SD) [2], Lovesey model (LO) [7], Wahnström and Sjögren mode-coupling theory (WS) [8], de Schepper and Ernst theory (dSE) [15], and Nelkin-Ghatak model (NG) [17].

lated data may also be observed in Fig. 3. This constrasts with the results of Torcini et al. [12], who found an excellent agreement between the $S_{s}(k, \omega)$ results calculated from the WS theory and their simulation data for liquid lithium at 470 K. This suggests that $F_{s}(k, t)$ provides a more refined test than $S_{s}(k, \omega)$ for theoretical models.

\section{Second memory function}

In order to understand why the Lovesey model shows such singular behavior and the reasons for the rather poor results obtained using the WS theory, a more accurate analysis based on the second memory function has been carried out. The normalized memory functions $N_{s}^{L O}(k, t), N_{s}^{W S}(k, t)$, and $N_{s}^{M D}(k, t)$ are plotted in Fig. 4. The MD results are similar to those obtained by Shimojo and co-workers for liquid sodium [14]. So, for all $k$ 's, $N_{s}^{M D}(k, t)$ takes positive values, decreases faster as $k$ is raised, and shows a maximum at $t$ $\sim 0.12$ ps for $k \leqslant 2.5 \AA^{-1}$. It should be emphasized that, for all wave numbers, $N_{s}^{W S}(k, t)$ shows a better agreement with the MD data than $N_{s}^{L O}(k, t)$. This is particularly true at short times where $N_{s}^{W S}(k, t)$ fits $N_{s}^{M D}(k, t)$ very well, in contrast to the too slow and exponential decay of $N_{s}^{L O}(k, t)$ which gives rise to the oscillations in $F_{s}^{L O}(k, t)$ observed in Fig. 3. Therefore, the rather good agreement between $F_{s}^{L O}(k, t)$ and 

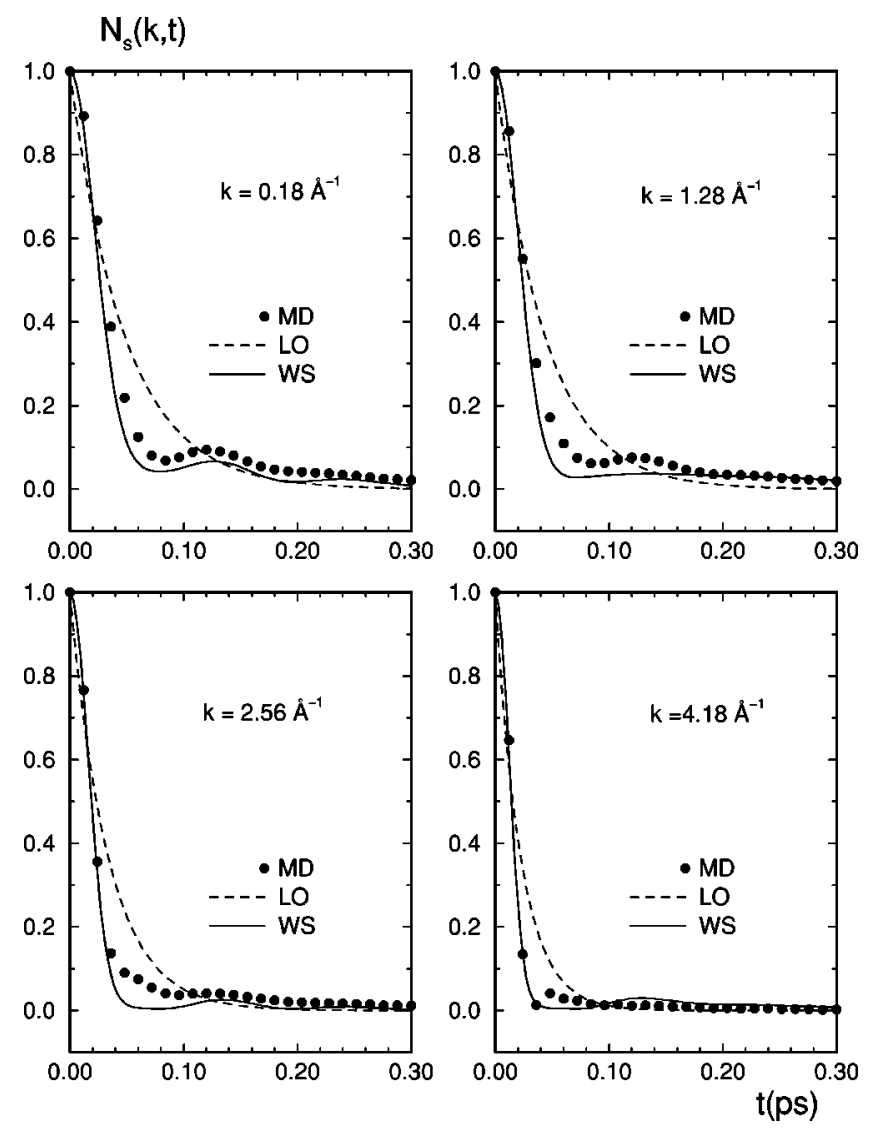

FIG. 4. Normalized values of the second memory function of $F_{s}(k, t)$. Simulation data (MD), Lovesey model (LO) [7], and Wahnström and Sjögren mode-coupling theory (WS) [8].

$F_{s}^{M D}(k, t)$ should be attributed to a cancellation of inaccuracies and cannot be considered as the result of a rigorous theory that properly includes the specific details of the individual atomic motion. Thus the WS theory seems more "realistic,' particularly for small wave numbers. However, for large $k$ 's and short times there are some discrepancies between the WS predictions and the MD results. This can be explained by bearing in mind that in the free particle limit, where $F_{s}(k, t)=e^{-\omega_{0}^{2} t^{2} / 2}, N_{s}(k, t)$ presents a minimum located at very short times. For this reason $N_{s}^{M D}(k, t)$ at $k$ $=4.18 \AA^{-1}$ exhibits a clear minimum at 0.04 ps. However, $N_{s}^{W S}(k, t)$, which has been calculated assuming the "Gaussian" Ansatz $N_{s B}^{\text {Gau }}(k, t)$, does not show this minimum. It is important to note that a minimum would appear (see Fig. 2) if the "kinetic'" approach $N_{s B}^{\text {Kin }}(k, t)$ were assumed for the binary term, which indeed satisfies the free particle limit. However, in this case the minimum is too deep and the agreement with the MD results is still worse than using $N_{s B}^{G a u}(k, t)$.

In order to analyze more carefully the discrepancies between $N_{s}^{M D}(k, t)$ and $N_{s}^{W S}(k, t)$ we have calculated

$$
\begin{aligned}
\Delta N_{s}(k, t) & =\frac{N_{s}^{M D}(k, t)-N_{s}^{W S}(k, t)}{N_{s}^{M D}(k, 0)} \\
& =\frac{N_{s}^{M D}(k, t)-N_{s B}^{G a u}(k, t)-N_{s M C}(k, t)}{N_{s}^{M D}(k, 0)} .
\end{aligned}
$$
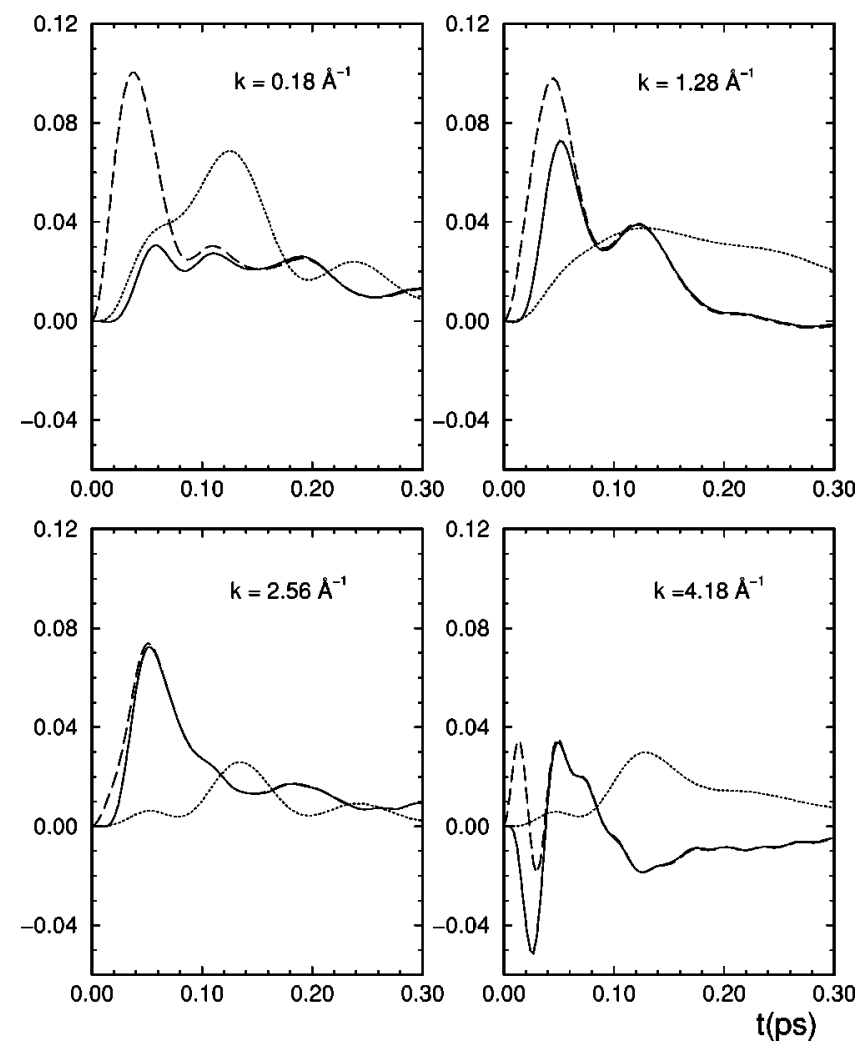

FIG. 5. Solid line $\Delta N_{s}(k, t)=\left[N_{s}^{M D}(k, t)\right.$ $\left.-N_{s}^{W S}(k, t)\right] / N_{s}^{M D}(k, 0)$ using the fitted $\tau_{G}^{f i t}(k)$ parameters (Table I); long dashed line, the same but with the theoretical $\tau_{G}^{\text {theo }}(k)$ parameters (Table I); dotted line, normalized mode-coupling term $N_{s M C}(k, t) / N_{s}^{M D}(k, 0)$.

The resulting $\Delta N_{s}(k, t)$ functions are shown in Fig. 5. For the sake of comparison the normalized mode-coupling contributions $N_{s M C}(k, t) / N_{s}^{M D}(k, 0)$ have also been plotted in Fig. 5. For small $k$ 's $N_{s M C}(k, t)$ is very similar to the modecoupling term of the velocity autocorrelation first memory function [20]. For all $k$ 's $N_{s M C}(k, t)$ exhibits a three peak structure with the peaks located at the same time position. It should be noted that the second peak of $N_{s M C}(k, t)$ gives rise to the $N_{s}^{M D}(k, t)$ maximum that may be observed in Fig. 4, at $t \sim 0.13$ ps.

$\Delta N_{s}(k, t)$ can be taken as the remaining term that should be included in the WS theory in order to obtain the "exact" MD result. However, as the time parameter $\tau_{G}(k)$ of Eq. (16) has been calculated approximately, an accurate test of the WS theory can be made only if the "correct" $\tau_{G}(k)$ parameter is used. To this end the difference between $N_{s}^{M D}(k, t)$ and $N_{s M C}(k, t)$ has been fitted to the Gaussian functional form given by Eq. (16). The $\tau_{G}(k)$ values obtained from the fitting and those calculated from Eq. (17) are compared in Table I. In Fig. 5 has also been plotted the difference $\Delta N_{s}^{f i t}(k, t)$ calculated using the fitted $\tau_{G}(k)$ values to compute $N_{s B}^{G a u}(k, t)$. For small and intermediate wave numbers $\Delta N_{s}^{f i t}(k, t)$ shows positive values that are of the same order of magnitude as or even larger than those of the mode-coupling term $N_{s M C}(k, t)$. At large $k$ 's $\Delta N_{s}^{f i t}(k, t)$ shows a deep negative minimum at short times, which again 
TABLE I. Theoretical and fitted time parameters $\tau_{G}(k)$.

\begin{tabular}{ccc}
\hline \hline$k\left(\AA^{-1}\right)$ & $\tau_{G}^{\text {theo }}(k)(\mathrm{fs})$ & $\tau_{G}^{f i t}(k)(\mathrm{fs})$ \\
\hline 0.185 & 31.40 & 35.50 \\
1.28 & 28.42 & 30.45 \\
2.56 & 22.81 & 23.28 \\
4.18 & 17.04 & 18.42 \\
\hline \hline
\end{tabular}

illustrates the inadequacy of the "Gaussian" approach. Moreover, at long times $\Delta N_{s}^{f i t}(k, t)$ takes negative values and it looks like the mirror image of $N_{s M C}(k, t)$. This suggests that the WS theory overemphasizes the mode coupling contribution at large $k$ 's. The results of previous studies on the velocity autocorrelation function $[20,40]$ and the self scattering function $[13,14,21]$ suggested that the decomposition of the memory function into two independent terms is unrealistic. Our results support this idea, since the largest $\Delta N_{s}^{f i t}(k, t)$ values correspond to the time interval for which $N_{s B}^{G a u}(k, t)$ comes close to zero (see Fig. 2) and $N_{s M C}(k, t)$ starts to rise. It should be emphasized that the corrective terms that should be added to the WS results are of the same order of magnitude as the mode-coupling contributions.

\section{CONCLUSIONS}

We have compared the $F_{s}(k, t)$ data calculated by MD with the results obtained using the models and theories of simple diffusion [2], Nelkin-Ghatak [17], Lovesey [7], de Schepper and Ernst [15], and Wahnström and Sjögren [8]. We have found how the predictions obtained using the Wahnström and Sjögren theory and the Lovesey model show the best agreement with the simulation data at different wave numbers. However, there are noticeable discrepancies between the results from MD and those from these theoretical approaches. $F_{s}^{W S}(k, t)$ decays too fast, specially at low $k$, whereas $F_{s}^{L O}(k, t)$ exhibits some nonrealistic oscillations, which are more marked at high $k$. A detailed analysis based on calculation of the $N_{s}(k, t)$ memory functions suggests that the predictions of the Wahnström and Sjögren theory are more realistic than those of the Lovesey model.

Following the Wahnström and Sjögren scheme, $N_{s}^{W S}(k, t)$ was computed as the sum of a binary and a mode-coupling term. Two different Ansätze for the binary term have been compared with the binary term obtained from the simulation. We found a good quantitative agreement between the results obtained using the "Gaussian" approach and the "simulation" results for all wave numbers. However, at large $k$ the binary function calculated from the simulation exhibits damped oscillations which are not reproduced by the "'Gaussian"' approach. On the other hand, the "kinetic" Ansatz, which satisfies the free particle limit condition, overestimates the oscillatory behavior for intermediate and large k's.

Our findings suggest that an improvement of the WS theory would require the use of a more refined binary term. Moreover, the separation of the memory function into two independent parts corresponding to the binary and modecoupling terms seems rather unrealistic. So a modified version of the WS theory that incorporates a certain degree of interdependence between the binary and mode-coupling terms could significantly improve this theoretical approach.

\section{ACKNOWLEDGMENTS}

The authors thank Umberto Balucani for useful suggestions. Financial support from the DGICYT of Spain (Grant No. PB96-0170-C03-02) and the CIRIT of the Generalitat de Catalunya (Grant No. 1999SGR-00146) is also acknowledged.
[1] P.A. Egelstaff, An Introduction to the Liquid State (Clarendon Press, Oxford, 1992).

[2] J.P. Hansen and I.R. McDonald, Theory of Simple Liquids (Academic, London, 1986).

[3] J.P. Boon and S. Yip, Molecular Hydrodynamics (McGrawHill, New York, 1980).

[4] U. Balucani and M. Zoppi, Dynamics of the Liquid State (Clarendon Press, Oxford, 1994).

[5] M.P. Allen and D.J. Tildesley, Computer Simulation of Liquids (Clarendon Press, Oxford, 1987).

[6] P.H.K. de Jong, P. Verkerk, and L.A. de Graaf, J. Phys.: Condens. Matter 6, 8391 (1994).

[7] S.W. Lovesey, J. Phys. C 6, 1856 (1973).

[8] G. Wahnström and L. Sjögren, J. Phys. C 15, 401 (1982).

[9] A. Sjölander, in Amorphous and Liquid Materials, edited by E. Lüscher (Nijhoff, Dordrecht, 1987).

[10] U. Balucani, A. Torcini, and R. Vallauri, Phys. Rev. B 47, 3011 (1993).

[11] J. Casas, D.J. González, and L.E. González, Phys. Rev. B 60, 10094 (1999).
[12] A. Torcini, U. Balucani, P.H.K. de Jong, and P. Verkerk, Phys. Rev. E 51, 3126 (1995).

[13] W. Gudowski, M. Dzugutov, and K.E. Larsson, Phys. Rev. E 47, 1693 (1993).

[14] F. Shimojo, K. Hoshino, and M. Watabe, J. Phys. Soc. Jpn. 63, 1821 (1994).

[15] I.M. de Schepper and M.H. Ernst, Physica A 98, 189 (1979).

[16] P. Verkerk, Nuovo Cimento D 12, 441 (1990).

[17] M. Nelkin and A. Ghatak, Phys. Rev. 135, A4 (1964).

[18] Handbook of Mathematical Functions, edited by M. Abramovitz and I.A. Stegun (Dover, New York, 1972).

[19] L.E. González, D.J. González, and M. Canales, Z. Phys. B 100, 601 (1996).

[20] M. Canales and J.A. Padró, J. Phys.: Condens. Matter 9, 11 009 (1997).

[21] U. Balucani, A. Torcini, A. Stangl, and C. Morkel, Phys. Scr. 57, 13 (1995).

[22] J.L. Lebowitz, J.K. Percus, and J. Sykes, Phys. Rev. 188, 487 (1969).

[23] D. Levesque and L. Verlet, Phys. Rev. A 2, 2514 (1970). 
[24] M.S. Jhon and D. Forster, Phys. Rev. A 12, 254 (1975).

[25] L.E. González, D.J. González, M. Silbert, and J.A. Alonso, J. Phys.: Condens. Matter 5, 4283 (1993).

[26] D. Beeman, J. Comput. Phys. 20, 130 (1976).

[27] M. Canales, J.A. Padró, L.E. González, and A. Giró, J. Phys.: Condens. Matter 5, 3095 (1993).

[28] M. Canales, L.E. González, and J.A. Padró, Phys. Rev. E 5, 3656 (1994).

[29] H. Sinn and E. Burkel, J. Phys.: Condens. Matter 8, 9369 (1996)

[30] H. Sinn, F. Sette, U. Bergmann, Ch. Halcoussis, M. Krisch, R. Verbeni, and E. Burkel, Phys. Rev. Lett. 78, 1715 (1997).

[31] T. Scopigno, U. Balucani, A. Cunsolo, C. Masciovecchio, G. Ruocco, F. Sette, and R. Verbeni, Europhys. Lett. 50, 189 (2000).
[32] B.J. Berne and G.D. Harp, Adv. Chem. Phys. 17, 216 (1970).

[33] L. Verlet, Phys. Rev. 165, 201 (1968).

[34] G. Zerah, J. Comput. Phys. 61, 280 (1985).

[35] C. Hoheisel and R. Vogelsang, Comput. Phys. Rep. 8, 1 (1988).

[36] W.H. Press, B.P. Flannery, S.A. Teukolsky, and W.T. Vetterling, Numerical Recipes (Cambridge University Press, New York, 1989).

[37] S.W. Lovesey, J. Phys. C 4, 3057 (1971).

[38] K. Hoshino, H. Ugawa, and M. Watabe, J. Phys. Soc. Jpn. 61, 2182 (1992).

[39] D.J. González, L.E. González, and K. Hoshino, J. Phys.: Condens. Matter 6, 3849 (1994).

[40] K.E. Larsson, J. Phys.: Condens. Matter 6, 2835 (1994). 